\title{
Automatic Laser Pointer Detection Algorithm for Environment Control Device Systems Based on Template Matching and Genetic Tuning of Fuzzy Rule-Based Systems
}

\author{
Chávez F. ${ }^{1}$ Fernández F. ${ }^{1}$ Gacto M.J. ${ }^{2}$ Alcalá R. $^{3}$ \\ ${ }^{1}$ University of Extremadura, Department of Computer Science \\ Santa Teresa de Jornet 38 , \\ Mérida, 06800, Spain \\ E-mail: \{fchavez, fcofdez\}@unex.es \\ ${ }^{2}$ University of Jaén, Department of Computer Science, \\ Campus Las Lagunillas, $s / n$ \\ Jaén, 23071, Spain \\ E-mail:mgacto@ujaen.es \\ ${ }^{3}$ University of Granada, Department of Computer Science and Artificial Intelligence, \\ Research Center on Information and Communications Technology, \\ C/ Periodista Daniel Saucedo Aranda $s / n$ \\ Granada, 18071, Spain \\ E-mail:alcala@decsai.ugr.es \\ Received 15 December 2010 \\ Accepted 1 June 2011
}

\begin{abstract}
In this paper we propose a new approach for laser-based environment device control systems based on the automatic design of a Fuzzy Rule-Based System for laser pointer detection. The idea is to improve the success rate of the previous approaches decreasing as much as possible the false offs and increasing the success rate in images with laser spot, i.e., the detection of a false laser spot (since this could lead to dangerous situations). To this end, we propose to analyze both, the morphology and color of a laser spot image together, thus developing a new robust algorithm. Genetic Fuzzy Systems have also been employed to improve the laser spot system detection by means of a fine tuning of the involved membership functions thus reducing the system false offs, which is the main objective in this problem. The system presented in this paper, makes use of a Fuzzy Rule-Based System adjusted by a Genetic Algorithm, which, based on laser morphology and color analysis, shows a better success rate than previous approaches.
\end{abstract}

Keywords: Interaction Systems, Fuzzy Rule-Based Systems, Genetic Fuzzy Systems, Laser Pointer Detection, Domotic Control Systems

\section{Introduction}

Nowadays, a home can be controlled by different devices like special indoor control devices, or by means of mobile phones, personal computers, etc. using the Internet if the users are outdoors. Recent research in intelligent homes has provided valuable 
solutions for controlling home devices ${ }^{12},{ }^{17},{ }^{39}, 54$, 55,56 .

We can find different kinds of smart homes systems in the specialized literature. Park et al. ${ }^{39}$ presented a robotic smart house, by means of which disabled people can be assisted by a robot sending orders with body movements. Other researchers have used non-invasive brain-computer interfaces ${ }^{17}$, where a brain computer interface is used to control different devices.

Additionally, the work presented in ${ }^{57}$ makes use of a robot to help disabled people to pick up different objects. In this work, the authors also proposed an interesting way to point out the desired objects by means of a laser pointer. The robot should then be able to detect the laser spot on an object in order to pick this object up. Since they represent cheap and easy to handle devices, laser pointers have been used as an indicator element for controlling large displays ${ }^{28},{ }^{36},{ }^{34},{ }^{47}$ and now they have also been used in the environment control system.

The main goal in these types of systems is to detect the laser spot effectively. In ${ }^{57}$, the authors deal with this problem by using special physical filters in the video camera while it is taking the environment photos, with the aim of only capturing the laser spot. However, this is still an open problem that can be addressed by using laser spot detection algorithms based on the original non-filtered images, avoiding the expense of using special cameras and filters.

An alternative, using standard and therefore cheaper cameras, can be found in ${ }^{14}$ where a set of algorithms, were proposed to detect the laser spot effectively on the image obtained by a standard video camera. Nevertheless, this algorithm presents some false offs, when a laser spot is detected by the algorithm but the image does not have any laser spot. In this event, a wrong order is sent to the domotic system which could provoke undesirable, dangerous or at least unexpected, situations. For this reason, in 16 we presented a new approach hybridizing a classic technique with the use of a Fuzzy Rule-Based System (FRBS) designed using expert knowledge ${ }^{3}, 45$, 46 to try to improve the success rate in images without laser spots. Further, this initial FRBS was tuned in ${ }^{15}$ by means of a Genetic Algorithm (GA), in or- der to improve the success rate in images without laser spots.

In this paper, we present a new approach to detect the laser spot in the environment device control system presented in ${ }^{15}$ and ${ }^{16}$ by considering the color influence in laser spot detection and using a simple automatic Rule Base (RB) generation. This method solves the problems that the approaches proposed in 15 and ${ }^{16}$ present when dealing with new situations as a consequence of extending the set of available example images from 105 in ${ }^{15},{ }^{16}$ to 990 . This new set of example images represents an almost complete range of different light and distance conditions in their real use by humans, and it allows us to consider a cross fold validation approach in order to adequately validate the proposed approaches. In fact, we can observe how the results obtained by the previous approaches are not able to overcome those obtained by the classic techniques ${ }^{14}$. The main reason is that these approaches only consider information about the morphology of the laser spot but not also necessary information about the color and energy.

For this reason, we propose a new hybrid algorithm based on classic techniques such as Template Matching (TM) and FRBS (TM+FRBS) with 3 new variables corresponding to the color of the laser spot in the Red, Green and Blue color format known as the RGB color system. Since it involves a significant increase in the number of variables and the generation of a new set of rules for the FRBS, we apply a simple automatic rule generation method ${ }^{58},{ }^{59}$ considering the extended set of images and following a cross validation approach instead of trying an expert based generation as in ${ }^{15}$ and then in ${ }^{16}$. With this new approach, we analyze the morphology and the color of the laser spot images, making it more precise than the previous approaches.

This contribution is arranged as follows. In section 2, the environment device system for environment control by using a laser pointer is described together with the previous approaches applied to solve this problem. Section 3 describes the proposed technique using the color influence and the automatic rule generation. The results obtained by the proposed technique are analyzed and compared to the previous proposals in Section 4. Section 5 presents 
some conclusions.

\section{Preliminaries: Laser pointers, system description and previous approaches}

This section introduces some preliminary approaches using a laser pointer as an interaction device. First, a short review of the previous systems which use laser pointers as a pointer device is presented. Second, the environment control device system with its different sections is described. Finally, the previous approaches used to solve the addressed problem are introduced in order to better situate our new proposal.

\subsection{Laser Pointer as a Pointer Device}

Usually, the works where the laser pointer has been used as pointer device, use the laser pointer on a large display and/or on systems to control smart homes and help disabled people.

The papers presented in ${ }^{2},{ }^{9},{ }^{10},{ }^{11},{ }^{19},{ }^{28},{ }^{29},{ }^{33}$, ${ }^{34},{ }^{36},{ }^{42},{ }^{44},{ }^{47}$ use a laser pointer as a pointing device on large displays. The aim of these works is to be able to control the different objects presented on a large display. The laser pointer was used in the same way as a mouse cursor. Thanks to the laser pointer, users can interact directly with objects projected on to the screen.

Different techniques have been used to solve the problem and detect the laser spot, such as threshold value, pattern recognition, color analysis, etc. Kirstein and Müller used an algorithm divided into three phases for detecting the laser spot. The algorithm phases were, Motion Detection, Pattern Recognition and Histograms Comparison ${ }^{29}$. By means of these techniques, the laser spot is detected in only $50 \%$ of the frames.

The works presented in ${ }^{2},{ }^{10},{ }^{11},{ }^{19},{ }^{31},{ }^{34},{ }^{36}$, ${ }^{47}$ used an algorithm only based on threshold value. These algorithms can classify the brightest pixels using a threshold value calculated previously. This set of classified pixels could be the laser spot pixels, but these algorithms have similar results to the algorithm referred to above.

A different technique for detecting the laser spot is presented in ${ }^{28}$. This algorithm uses different color systems, RGB and Hue, Saturation and Intensity (HSI) systems, for detecting the laser spot on an image. A video-camera takes a color image in the RGB system. The algorithm changes the color system to the HSI system. By means of a piecewisedefined function, the laser spot is detected.

The main problem in these previous works is that light conditions, inclinations and textures are controlled and fixed. For example, the laser spot can not be detected correctly with a high brightness light on the displays.

On the other hand, since the previous approaches typically present many false offs, special cams including optical filters have also been used. In ${ }^{19}$ the authors used wavelength bandpass filters together with several video-cameras to detect the laser spot. In ${ }^{11}$ and ${ }^{47}$ a red filter is applied to the videocamera to try to solve the false off problem. With the Homography technique, several video-cameras and several computers are used in order to to detect the correct laser spot position ${ }^{2}$. In ${ }^{28}$ a laser filter is used to improve the efficiency of the algorithm. Finally, in ${ }^{10}$ and ${ }^{36}$ a set of images is analyzed and compared to try to eliminate the false offs. We observe that the laser spot is located not only by software, the authors use special filter cameras, several computers, and other intrusive techniques to detect the laser spot. Furthermore, in most cases the algorithms work in controlled light environment conditions. In this setting, it is easier to locate the laser spot than in a real home environment, where the brightness of light conditions are almost impossible to control, and the algorithms have to solve uncontrollable situations.

Recent research has attempted to integrate a domotic system in the home to help disabled and elderly people. These are known as smart homes ${ }^{6},{ }^{13}$, ${ }^{14},{ }^{17},{ }^{25},{ }^{27},{ }^{32},{ }^{37},{ }^{38},{ }^{39}$. The most relevant work is found in ${ }^{13}$, in which a robot picks up the object indicated by the user with a laser pointer. This is a similar technique to the technique presented in this paper, but the robot uses special physical filters in the video camera to filter the images and detect the laser spot. 


\subsection{Environment device control system}

In this subsection the environment device control system addressed in this paper is described. The system is divided into three sections, Family Tool Section, Algorithms Analyzer and Domotic Control System. Figure 1 shows the different system sections. In the following subsections we describe in detail each system section.

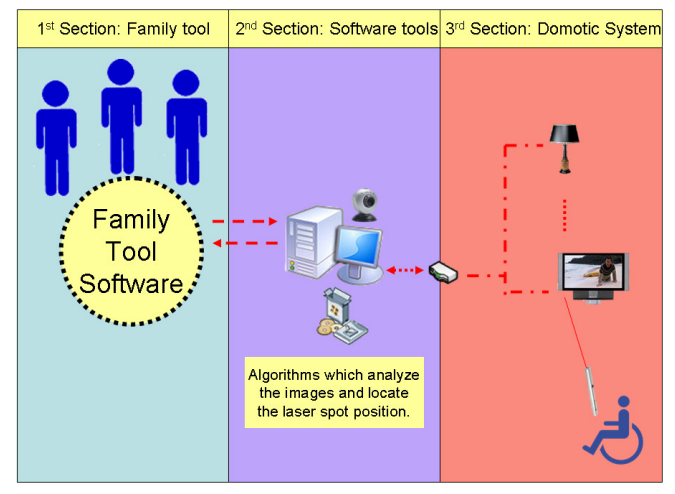

Fig. 1. Environment control system

\subsubsection{Family tool}

To start using the system, the users have to indicate where the different devices are, which will be handled by the domotic system. By means of the Family tool, a fixed video camera in a home environment takes an image and sends it to the computer. This image is shown and the user marks, by using a simple click method, the devices that will be handled by the domotic system. The subsequent marked image is known as an active zone. Therefore, an active zone is a set of pixels which contains information on the positioning of the devices. Previously, the domotic system must be configured by an expert, who will indicate how many home devices will be handled and the type of each device. When the active zone has been marked, the user must select a device from the list of devices previously defined by the domotic system expert. With this action, the active zone will be matched with the home device and the system will have information on the positioning of this device.
Once the active zones have been marked, we can indicate the device that we want to use by a laser pointer. The system will analyze the actives zones previously indicated, and if the laser spot is found in an active zone, the domotic system will handle the associated home device.

Finally, if a home device or camera is changed within a given environment then the active zones must be recreated so that they are updated by the system.

\subsubsection{Algorithms Analyzer}

The second section of the system is the algorithm which will analyze the images sent by the fixed video camera and locate the position of the laser spot. By means of the laser pointer the user selects the device he wants to use. The laser pointer makes a red or green spot on the device. The video camera sends the environmental image with the laser spot information and the computer analyzes this image and locates the laser spot. If the laser spot is in an active zone, the user wants to use the device marked in this zone, and finally, the computer sends the necessary orders to turn on/off this device using the domotic system.

The video camera used by the system is an Axis $207 \mathrm{~W}$ video camera. This camera has $1.3 \mathrm{Mega}-$ pixels with an image sensor of $1 / 3$ " Micron progressive RGB CMOS, lens dimension of $3.6 \mathrm{~mm} / \mathrm{F} 1.8$ and $2-10000$ (Lux). The laser pointer used is a green laser pointer class III with maximum power $<5 \mathrm{~mW}$ and wave length of $532 \mathrm{~nm}$.

In some of our previous works, a number of algorithms aiming at detecting the laser spot in the images sent by the camera were employed and analyzed ${ }^{14},{ }^{16}$ and ${ }^{15}$. These algorithms are presented in the following section.

\subsubsection{Domotic Control System}

The third section of the system deals with the devices controlled by the domotic system using $\mathrm{KNX/EIB}$ architecture ${ }^{23}$. Once the laser spot is found and it is in an active zone, the system sends different orders to turn on/off the device associated with this active zone, to the domotic system. But 
the system can be configured to do more complex actions. Depending of the final user, the complex functions of the home devices can be encoded differently. For instance, if the final user is a person with a physical disability, a panel will be place close to the device. This panel could have four sections, Volume, Channel, + and -, indicating to the user the complex action for the TV on the panel. Another possibility could be to include different active areas in a single device, allowing a larger set of actions.

\subsection{Previous approaches applied to this problem}

This contribution is focused on the $2^{\text {nd }}$ Section of the environment device control system explained in the previous subsection, in order to improve the laser spot detection ability, which becomes one of the most important tasks in these kinds of systems. The algorithms previously used to detect the laser spot on an image have been the following:

1. Dynamic Umbralization (DU)

2. Template Matching (TM)

3. Template Matching + Dynamic Umbralization $(T M+D U)$

4. Template Matching + FRBS design from Expert's Experience $\left(T M+F R B S_{\text {expert }}\right)$.

The home environment control system based on a laser pointer presented in ${ }^{14}$ used three different classic algorithms, DU, TM and TM + DU. In ${ }^{16}$ an initial hybrid model is proposed combining TM with an FRBS obtained from expert experience, which is later improved by applying a first version of a genetic tuning of the FRBS Membership Functions (MFs) in ${ }^{15}$. In the following subsections, we will briefly introduce the methods that we have presented in this previous work to detect the laser spot on an image (a deeper description of these algorithms can be found in $\left.{ }^{14},{ }^{15},{ }^{16}\right)$.

\subsubsection{Dynamic Umbralization}

DU algorithm ${ }^{14}$ is based on umbralization ${ }^{41}$, which is a technique frequently used to locate information on an image using a threshold value.
This technique calculates a value known as a threshold value. This value is used to eliminate the image information over or under it. With this technique, the relevant information of an image can be separated; that is, the laser spot information.

The steps we took in the procedure are the following. We analyzed a set of images with and without laser spot pixels, and discovered that the laser spot pixels have their own characteristic information. When these pixels were transformed to numerical information, we observed that the numerical value of these pixels was a long negative number, however, the rest of the pixels had greater numerical values. We used a static threshold value which could eliminate all pixels without laser spot information. The results were positive; the algorithm could locate the laser spot on the image. The next step was to change the light conditions. Depending on different light conditions the threshold value calculated was not the ideal. Therefore, we calculated a dynamic threshold value using four parameters extracted from the image. The parameters taken from the histogram included the largest value, a percentage of the largest value and the average and the sum of the pixel numerical information, thereby giving us a more dynamic umbralization. With these parameters we could calculate a different threshold for each image sent by the video camera.

The process to calculate the threshold value is as follows:

- The first step is to calculate the histogram. This histogram is calculated using a whole image in color sent by the video camera. For each pixel, the algorithm takes the integer value of the pixel and this value is divided by 256 . The algorithm uses a vector with 256 positions, the vector position corresponding with the remainder of the division between the integer value of the pixel and 256 , is increased by 1 . Once all pixels have been divided by 256 the system obtains wath we call, the histogram of the image. Once the histogram has been calculated, the system obtains the first parameter, the largest histogram value.

- The second step is to calculate the percentage of the largest image value. The system obtains the largest integer value of the image and calculates 
Chávez F., Fernádez F., Gacto M.J., Alcalá R.

Table 1. Parameters balance

1 The sub interval balance is obtained by applying the criteria in Table 2 for each parameter.

\begin{tabular}{lc}
\hline Parameter & Balance \\
\hline \% largest value of the histogram & $4 *$ sub interval balance $(1)$ \\
Largest value of the histogram & $3 *$ sub interval balance $(1)$ \\
Average & $2 *$ sub interval balance $(1)$ \\
Sum & $1 *$ sub interval balance $(1)$ \\
\hline
\end{tabular}

the percentage, using the expression (1).

- The third step is to calculate the average of the image integer values.

- The fourth step is to calculate the sum of the image integer values.

$$
V_{u m b}=\left(\left(\left(S v \_I_{\text {max }}\right)-\left(S v \_I_{\text {min }}\right)\right) * \frac{X}{20}\right)+\left(S v \_I_{\text {min }}\right)
$$

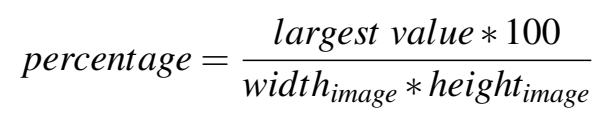

In ${ }^{14}$ the minimum and maximum integer values are presented, average and the sum value that an image can obtain. These value are divided into six subintervals as we can see in ${ }^{14}$. For each parameter, the algorithm calculates the intervals wherever it is. The parameter values are of varying importance within the dynamic umbralization calculation. The importance of this is shown in Table 1.

Once the intervals where the different parameters had been calculated, we divided the intervals into three different sub intervals and checked where each parameter value was within these three sub intervals. In order to find where the parameter value was within the sub intervals, we established Table 2:

Table 2. Sub intervals balance

\begin{tabular}{lc}
\hline Level & Balance \\
\hline Low $(0 \%-33 \%)$ & 0 \\
Middle(33\%-66\%) & 1 \\
High(66\%-100\%) & 2 \\
\hline
\end{tabular}

The threshold value is obtained in the final step as follows: we must take the smallest interval obtained from all parameters used $\left(I_{\min }\right)$ and the largest interval obtained from all parameters used $\left(I_{\max }\right)$. With these two numbers we can obtain the rate of umbralization as we can see in Table 3.

The final threshold value is calculated by applying the following expression.

Where $X$ is the sum of the balance parameters obtained when applying table $1, S v I_{\min }$ is the lowest value of $I_{\min }$ intervals corresponding to table 3 and $S v I_{\max }$ is the highest value of $I_{\min }$ intervals corresponding to the same table.

The threshold value is calculated for each image sent by the video camera. With this operation the threshold value changes in real time, depending on the light conditions.

The DU algorithm eliminates all pixels under the $V_{u m b}$ calculated. The pixels which have not been eliminated are the laser spot pixels.

However, we have to point out that in nonoptimum light conditions and in images with reflections, the DU algorithm had false offs. Figure 2 shows an example of a laser spot and a false off located.

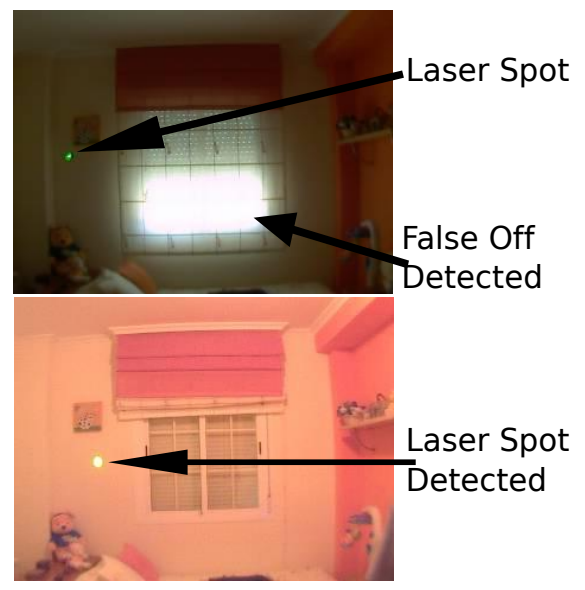

Fig. 2. False off and success using the DU algorithm. 
Automatic Laser Pointer Detection Algorithm using Template Matching and Fuzzy Rule-Based Systems

Table 3. Umbralization intervals

\begin{tabular}{lccccc}
\hline Interval 0 & Interval 1 & Interval 2 & Interval 3 & Interval 4 & Interval 5 \\
0 & $2 * 10^{6}$ & $4 * 10^{6}$ & $6 * 10^{6}$ & $8 * 10^{6}$ & $10 * 10^{6}$ \\
$2 * 10^{6}$ & $4 * 10^{6}$ & $6 * 10^{6}$ & $8 * 10^{6}$ & $10 * 10^{6}$ & $12 * 10^{6}$ \\
\hline
\end{tabular}

Trying to eliminate the false off we used the TM technique. Instead of analyzing the pixel information, this technique tries to find a template laser previously calculated on the image sent by the video camera.

\subsubsection{Template Matching}

This technique is based on locating a laser template which is on an image ${ }^{14}$. This algorithm analyzes small image sections that are compared with the template which it wants to find. In this operation the correlation between the image section and the template is calculated. Using the pattern search technique ${ }^{41}$ the algorithm can find a template for the whole image. Figure 3 shows a template laser used by the algorithm.

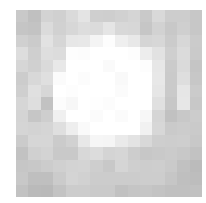

Fig. 3. Template laser spot

The kind of measure used to calculate the correlation values between the template and the image is known as Zero Mean Normalized Cross Correlation (ZMNCC). The value obtained with this kind of correlation is normalized between -1 and $1^{21}$ and ${ }^{35}$. The expression is as follows:

$$
\begin{gathered}
\Phi\left(I_{r}, I_{l}\right)=\frac{\sum_{i, j \in[-w, w]} A B}{\sqrt{\sum_{i, j \in[-w, w]} A^{2} \sum_{i, j \in[-w, w]} B^{2}}} \\
A=I_{r}(x+i, y+j)-\overline{I_{r}(x, y)} \\
B=I_{l}\left(x^{\prime}+i, y^{\prime}+j\right)-\overline{I_{l}\left(x^{\prime}, y^{\prime}\right)}
\end{gathered}
$$

where the expression part known as A contains the set of pixels which are in the principal image sec- tion, the section known as B contains the set of laser template pixels, and $\mathrm{w}$ is half the size of the window used.

In order to use this technique, a set of templates must be previously calculated. The algorithm to calculate the set of templates is divided into two sections:

1. Section 1: The algorithm takes 30 images of a laser spot on a white surface.

2. Section 2: The algorithm calculates an average image with the images previously taken.

To calculate the set of templates it is necessary to have a video camera fixed in a specific location and focused on a white surface. A laser spot is then drawn on this surface with a laser pointer. A snapshot image is then taken every 0.5 seconds, ending with a total of 30 images. With these 30 images the system calculates an average image in the second step. With the average image we eliminate the CCD noise from the video camera. This average image is the resulting template. This process is carried out between 2 and 5 meters from the video camera to the surface and with different light conditions. Once the process has finished, we obtain the set of templates used by the TM algorithm to search the laser spot in the images sent by the video camera.

The TM algorithm consists of a process to calculate the correlation value between a section of the images sent by the video camera with a similar size to the templates used. The system uses different templates depending on the environmental conditions. There are two parameters to chose the correct template; the distance and the light condition. The distance between the video camera and the home devices is known by the system, because for each zone, the user has to indicate the distance between the camera and the home devices. The light conditions depend on the system operating hours and a luminance image analysis. 
Using the expression (3) the TM algorithm obtains the correlation between an image section and the template used. Using a convolution technique the algorithm can analyze the whole image sent by the video camera using the correct template. Finally, all correlation values are saved in a vector together with the analyzed image coordinates, thereafter extracting the highest value. Generally, the template which is searched for can be found in the coordinates which have the highest values.

TM obtains better results than DU, however the new algorithm now has new false offs ${ }^{14}$. This is due to the fact that it may interpret small component devices like buttons, leds, circular reflections on devices, etc, as laser spots. An example of a false off obtained with the TM algorithm can seen in Figure 4.

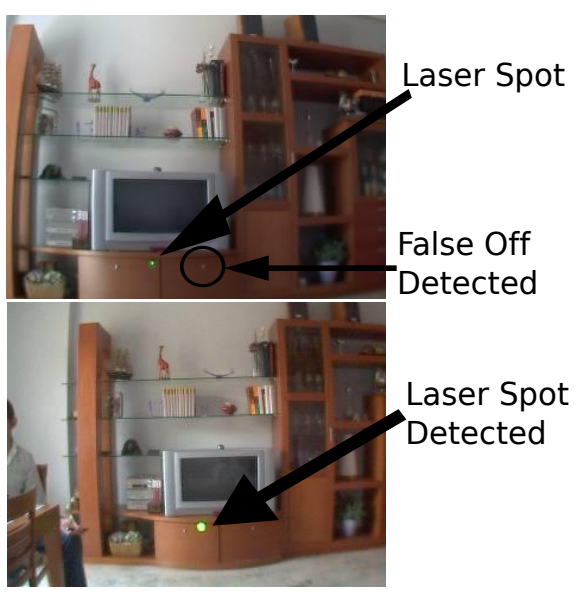

Fig. 4. False off and success using the TM technique

To eliminate false offs in both algorithms we developed a new algorithm to join the two techniques.

\subsubsection{Combining Template Matching \& Dynamic Umbralization Techniques}

This method ${ }^{14}$ combines the advantages of TM and DU. This algorithm analyzes only the active zones. For each active zone it searches a laser template and obtains a maximum correlation value. A section of the active zone of $15 \times 15$ pixels is taken where the highest correlation value was found. This section has a similar size to the laser template used. In this new image the laser spot could be found, as a high energy output was necessary. In the following step we use DU to check these pixels. If they have high energy, we can say that the laser spot can be found here, because this section is very similar to a laser template. The process is shown in Figure 5.

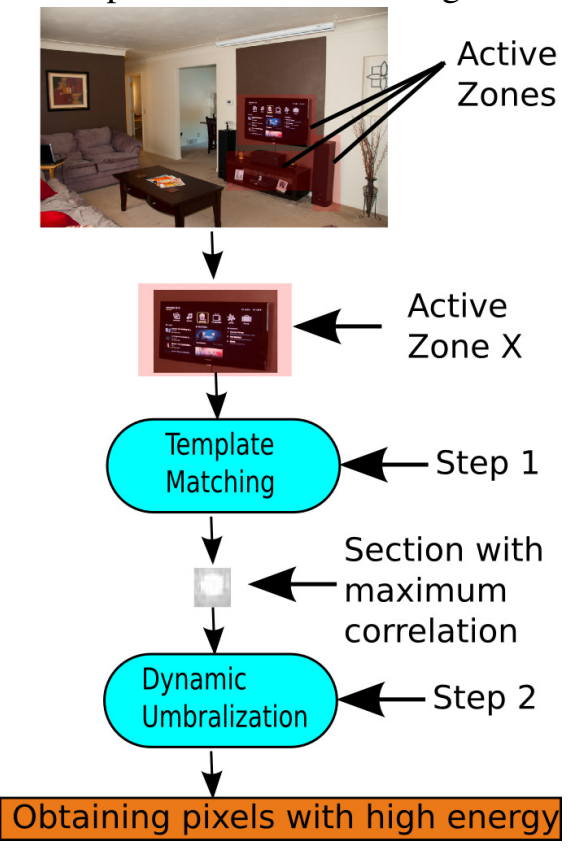

Fig. 5. TM + DU working scheme

\subsubsection{Combining Template Matching \& Fuzzy Rule-Based Systems}

In order to improve the results presented in ${ }^{14}$, an hybrid approach between TM and FRBSs has been proposed to determine whether an image section provided by TM is detected as a laser spot or not. This way of working allows us to have a system based on labels ${ }^{45},{ }^{46}$, with a near human language, making it easy to derive rules ${ }^{3}$ that can provide a positive or negative response for each analyzed image section.

Analyzing a laser template (see Figure 3), a set of interesting system variables were proposed in 16 by analyzing some example images, with and without laser spots. These variables determined by the expert (five inputs and one output) represent the following information: 
- X1: Long standard deviation.

- X2: Cross standard deviation.

- X3: Similarity to perfect circle value.

- X4: Laser spot number of pixels.

- X5: Percentile 80 value.

- Y: Laser spot probability (laser spot is detected if this value is over a threshold).

Once the input variables and their domains were defined, the expert defined useful rules and manually adjusted the MFs, definitions for the detection task. The process to determine these variables as well as the finally proposed FRBS from the expert's experience can be found in ${ }^{16}$.

The complete recognition task is therefore comprised of two main parts (algorithms). The first algorithm, TM, analyzes the image sent by the video camera together with a laser template image. This obtains the image section with the highest correlation. The obtained image section is then analyzed by using the FRBS proposed. This new combination of algorithms ${ }^{16}$, namely $T M+F R B S_{\text {expert }}$, directly presented a better performance than the algorithms described in sections 2.3.1 and 2.3.2 as we can see in ${ }^{16}$.

On the other hand, although the new approach, $T M+F R B S_{\text {expert }}$, presented better results than the previous techniques, it can be further refined by performing a genetic tuning of the MFs ${ }^{48}$, i.e., by means of a GA ${ }^{49},{ }^{50}$, the MFs of the FRBS are adjusted. This kind of hybridization between fuzzy $\operatorname{logic}{ }^{45},{ }^{46}$ and GAs is known as Genetic Fuzzy Systems (GFSs) ${ }^{51},{ }^{52},{ }^{53}$.

In this way a new approach, namely $T M+$ $F R B S_{\text {tuned GA }}$, was proposed in ${ }^{15}$ to perform an automatic evolutionary tuning of the initial FRBS based on the existence of 105 image samples that were used to train the model. Once the GA optimizes the MFs, parameters of the initial FRBS ( $\left.F R B S_{\text {expert }}\right)$, the new FRBS obtained ( $\left.F R B S_{\text {tuned } G A}\right)$ presented better results than the previous techniques used to detect the laser spot in the 105 images, as we can see in ${ }^{15}$.

\section{Improving the Hybrid Laser Pointer Detection Algorithm by Means of Color Detection and a Genetic Tuning of the obtained FRBS}

In order to ensure a good performance of the laser spot detection algorithm, we propose here a new technique to additionally use color and energy together with morphology of the laser spot by considering three new variables. Since this method involves an important change in the RB, which should consider this new information, a new set of rules is automatically generated by a simple RB generation method. Once the RB has been generated, a fine tuning process is applied to improve the system.

In the following section we propose a new FRBS model by including color detections with an automatic rule generation, in oder to obtain a more general FBRS with better results, trying to completely eliminate the false offs and to improve the success rate in images with laser spots. Then we describe the GA proposed to perform a fine tuning of the obtained FRBS.

\subsection{Extending the FRBS Model by Including Color Detection and Automatic Rule Generation}

The main problem for the environment control device system is the existence of false offs. The previous approaches introduced in section 2.3.4 create an increase in the false offs when the set of image samples is extended in order to consider similar to real conditions. In addition, the classic techniques work better than those approaches under these new conditions. Since these techniques only analyze the laser spot morphology, at this point we consider the introduction of color analysis, similar to the technique used in ${ }^{28}$ on large displays (in this work the environment conditions are controlled), where the authors only used color analysis. This technique is based on the RGB color system.

If we observe a laser spot image, we can see that there are special color characteristics. We use a green laser pointer, for this reason, the laser spot generated is green (see Figure 6). To be able to analyze the laser spot color, it is necessary to extract 
new parameters from the analyzed image. These parameters must be obtained using the red, green and blue color component of each pixel. The process to obtain the color parameters is the following: using the MatLab sentence rgb2ind - Convert RGB image to indexed image -, the image is represented in two colors, obtaining the predominant colors of the image. If the image is a laser spot image, we will obtain a transformed image with two colors near to the green tonality in the RGB color system. In other cases, the predominant tonality will be nearer to a gray color. In both cases, the system extracts the highest color tonality represented by three new variables, corresponding to the red, green and blue components (see Figure 7).

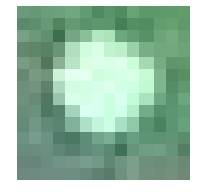

Fig. 6. Color laser spot

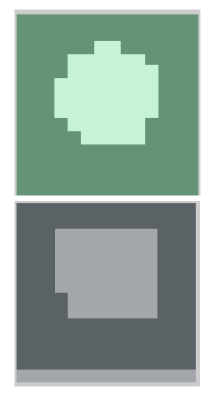

Fig. 7. Images processed

By means of a similar process used in the previous approach, the parameters X1, X2, X3, X4 and $\mathrm{X} 5$ are extracted. Finally, the values red, green and blue of the more predominant tonality of the transformed image are added to the input values for the FRBS. Introducing 3 new variables produces a significant increase in the number of rules needed for the FRBS. Due to the increase in the variables, there is an exponential increase in the work being carried out by the expert, making the generation of the FRBS by hand practically impossible. Therefore, it is necessary to use an automatic generation of the rules. This generation is based on applying the Wang and Mendel algorithm ${ }^{59}$, where from a set of data and an initial equidistant strong fuzzy partition with $5 \mathrm{MFs}$, it can automatically obtain a set of rules that will provide an initial solution for the problem.

The fuzzy rule set type used in this contribution is the following:

$$
\text { if } X_{1} \text { is } A_{1} \text { and ... and } X_{n} \text { is } A_{n} \text { THENY is } B
$$

where $X_{i}(Y)$ are the input (output) linguistic variables, $A_{i}(B)$ are the linguistic labels used in the input (output) variables.

The fuzzy inference system uses the center of gravity weighted by the matching strategy as a defuzzification operator and the minimum t-norm as implication and conjunctive operators ${ }^{18}$. More complete information on the inference process of FRBSs can be found in ${ }^{7},{ }^{20}$ and ${ }^{60}$, where FBRSs are explained in detail. The FRBS presented in this paper uses Mamdani-type rules and, as can be seen from the previous description, the defuzzification method is based on mode B: Defuzzification First, Aggregation After. An example of this kind of fuzzy inference system is shown in ${ }^{3},{ }^{4},{ }^{5}$ and ${ }^{22}$ for the control of a Heating, Ventilating and Air Conditioning System. FRBSs are used in processes such as, industrial control ${ }^{61}$, robotics control ${ }^{1}$ or control in communications ${ }^{8}$.

By following this approach, the proposal presented in this paper is based on the generation of an FRBS with 8 input variables and one output. They are:

- X1: Long standard deviation.

- X2: Cross standard deviation.

- X3: Similarity to perfect circle value.

- X4: Laser spot number of pixels.

- X5: Percentile 80 value.

- X6: Green value.

- X7: Red value.

- X8: Blue value.

- Y: Laser spot probability (laser spot is detected if this value is over a threshold).

In this way, the FRBS not only analyzes the morphological appearance of the image sent by the 
video camera, but also the information on the color of the image which is being analyzed. This last aspect, the predominant color in the image, is very important since it clearly differentiates the false off images from the real laser spot images. This new approach provides an important improvement in the decrease of false offs.

\subsection{Genetic Tuning of the obtained FRBS.}

With the aim of making an FRBS performs better, some approaches try to improve the preliminary Data Base (DB) definition, i.e., the definitions of the MFs, or the inference engine parameters once the RB has been derived ${ }^{51,52,53}$. Classically, due to the wide use of the triangular-shaped MFs, the tuning methods ${ }^{51,48}$ refine the three definition parameters that identify these kinds of MFs (see Figure 8).

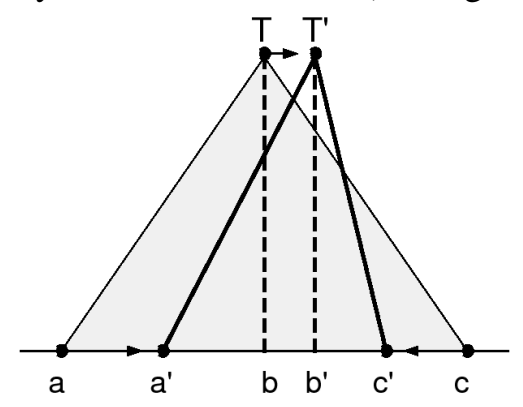

Fig. 8. Tuning by changing the basic MF parameters.

Among the different possibilities to perform tuning one of the most widely used approaches is the use of Evolutionary Algorithms to evolve the parameters' definitions. This is known as genetic tuning of MFs. A graphical representation of the tuning process is shown in figure 9 .

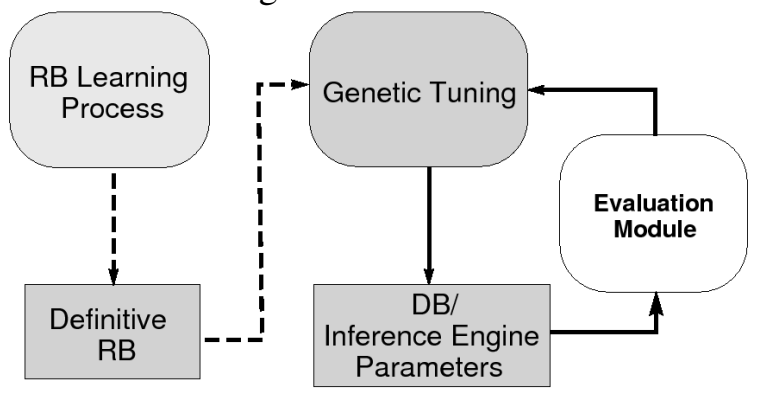

Fig. 9. Genetic tuning process.
To perform the genetic tuning we consider a GA that presents a real coding scheme and uses stochastic universal sampling as the selection procedure together with an elitist scheme. The operators employed to perform the individual recombination and mutation are uniform mutation and the max-minarithmetical crossover ${ }^{48}$. In the following, the components needed to design this process are explained.

\subsubsection{Chromosome Evaluation}

For each input example, the FRBS generates an output value into interval $[0,1]$. If this value is higher than a threshold value $(L)$ the example will be classified as a laser spot image, otherwise it will be classified as an image without a laser spot. Thus, an input example could be:

- False Negative (FN): If the example is classified as an image without a laser spot and it is a laser spot image.

- False Positive (FP): If the example is classified as a laser spot image and it is an image without a laser spot.

- Hit: If the example is correctly classified.

The objective of this algorithm is to minimize the number of FNs and FPs obtained by the FRBS. To evaluate a determined chromosome $C_{j}$ we use the following function:

$$
\text { Fitness }\left(C_{j}\right)=\frac{|F N|}{|D|}+3 \cdot \frac{|F P|}{|D|}
$$

where $|F N|$ is the number of FNs obtained, $|F P|$ is the number of FPs obtained and $|D|$ is the dataset size. Notice that the number of FPs is penalized in order to eliminate the wrong orders sent to the domotic system.

\subsubsection{Coding Scheme and Initial Gene Pool}

A real coding scheme is considered. Each chromosome is a vector of real numbers with size $3 \cdot F+1$ ( $F$ being the number of MFs in the given DB) in which the three parameters that identify each MFs 
and the threshold value are coded. Then, a chromosome $C_{j}$ has the following form, with $m^{i}$ the number of MFs of each of the $n$ variables in the DB:

$$
\begin{aligned}
& C_{j}=C_{j 1} C_{j 2} \cdots C_{j n} L_{j}, \\
& C_{j i}=\left(a_{j 1}^{i}, b_{j 1}^{i}, c_{j 1}^{i}, \ldots, a_{j m^{i}}^{i}, b_{j m^{i}}^{i}, c_{j m^{i}}^{i}\right), i=1, \cdots, n
\end{aligned}
$$

The initial gene pool is created making use of the initial DB definition. This initial DB with 0.5 as threshold value is encoded directly into a chromosome, denoted as $C_{1}$. The remaining individuals are generated at random in the variation intervals associated with each MF and to the threshold value. For each $M F_{f}=\left(a_{f}, b_{f}, c_{f}\right)$ where $f=(1, \ldots, F)$, the variation intervals are calculated in the following way (See Figure 10):

$$
\begin{aligned}
& {\left[I_{a_{f}}^{l}, I_{a_{f}}^{r}\right]=\left[a_{f}-\left(b_{f}-a_{f}\right) / 2, a_{f}+\left(b_{f}-a_{f}\right) / 2\right]} \\
& {\left[I_{b_{f}}^{l}, I_{b_{f}}^{r}\right]=\left[b_{f}-\left(b_{f}-a_{f}\right) / 2, b_{f}+\left(c_{f}-b_{f}\right) / 2\right]} \\
& {\left[I_{c_{f}}^{l}, I_{c_{f}}^{r}\right]=\left[c_{f}-\left(c_{f}-b_{f}\right) / 2, c_{f}+\left(c_{f}-b_{f}\right) / 2\right]}
\end{aligned}
$$

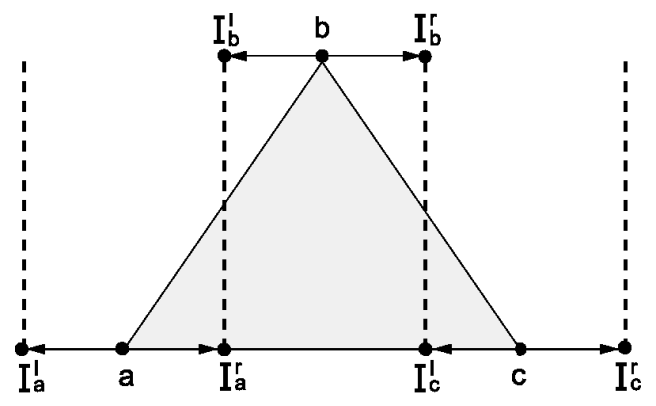

Fig. 10. The variation intervals.

The variation interval for the threshold value $(L)$ is $[0,1]$. Therefore, we create a population of chromosomes containing $C_{1}$ as its first individual and the remaining ones initiated randomly, with each gene in its respective variation interval.

\subsubsection{Max-min-arithmetical crossover}

If $C_{v}=\left(a_{v 1}^{1}, \ldots, e_{v k}, \ldots, L_{v}\right)$ and $C_{w}=$ $\left(a_{w 1}^{1}, \ldots, e_{w k}, \ldots, L_{w}\right)$ are to be crossed, the following four offspring are generated

$$
\begin{aligned}
& C_{1}=d C_{w}+(1-d) C_{v} \\
& C_{2}=d C_{v}+(1-d) C_{w} \\
& C_{3} \text { with } e_{3 k}=\min \left\{e_{v k}, e_{w k}\right\} \\
& C_{4} \text { with } e_{4 k}=\max \left\{e_{v k}, e_{w k}\right\}
\end{aligned}
$$

This operator can use a parameter $d(d=0.35)$ which is either a constant, or a variable whose value depends on the age of the population. The resulting descendants are the two best of the four offspring.

\subsubsection{Uniform mutation}

If $C_{j}=\left(a_{j 1}^{1}, \ldots, e_{j k}, \ldots, L_{j}\right)$ is a chromosome and the element $e_{j k}$ was selected for this mutation (the domain of $e_{j k}$ is $\left.\left[e_{j k}^{l}, e_{j k}^{r}\right]\right)$, the result is a vector $C_{j}^{\prime}=\left(a_{j 1}^{1}, \ldots, e_{j k}^{\prime}, \ldots, L_{j}\right)$ and

$$
e_{j k}^{\prime}=e_{j k}+\left(e_{j k}^{r}-e_{j k}\right) \cdot r,
$$

where $r$ is a random number into the interval $[-1.0,1.0]$.

\section{Framework and Experimental Study}

To evaluate the usefulness of the previous approaches and the new approach proposed in this paper, we have considered the environment control system presented in Section 2. In order to have a significant and well-established performance measure, we extend the 105 example images used in ${ }^{15}$ in order to complete a big data set containing 990 images, which represent a more complete range of working conditions. The images that make up the database were thus taken trying to simulate the final user actions in a large number of combinations of possible light and distance conditions:

- Light conditions: normal light, bright light and artificial light.

- Distance: distance between the laser pointer and the area where the device is placed. This distance is between 2 and 5 meters.

Each method studied in this paper uses the TM algorithm to obtain a candidate image section which could have the laser spot looked for. An image template previously calculated, using the technique shown in Subsection 2.3.2, is used to compare the 
image sent by the video camera with this template image, and thus, to extract the image section with the highest correlation using the TM algorithm. To extract this section, TM uses the most appropriate laser template, indicated by the expert, to look for the laser spot on an image. This process is used for the whole set of images.

This section is divided into three different parts:

- The first part describes the framework where the hybrid laser pointer detection algorithm is integrated into the domotic control system for real home environments.

- The second part describes the experimental setup used to run the studied algorithms.

- The third part shows the results obtained by the previous approaches $T M+D U, T M+F R B S_{\text {expert }}$ and $T M+F R B S_{\text {tuned } G A}$ compared to $T M+$ $F R B S_{R G B-\text { tunedGA }}$ in order to show the effectiveness of this new proposal with the new set of images. Finally, the influence of the color information consideration is studied, analyzing the results obtained by determining an appropriate precision value for the TM algorithm and by adapting the $\mathrm{RB}$ to the new set for examples considering the color variables.

\subsection{Framework: Domotic control used by the environment control system}

In this section we describe the framework of the domotic control system where the studied approaches are integrated to detect the laser spot on an image and to control the home device selected by the user. This system is endowed with KNX/EIB architecture ${ }^{23}$ by means of the laser pointer. Our software tool has been easily combined with KNX/EIB control software, such as ETS software ${ }^{30}$.

KNX technology is an open standard for all applications in home and building control. KNX technology is composed of BATIBUS, EIB y EHS, the base of $\mathrm{KNX}$ is EIB (European Installation Bus). $\mathrm{KNX/EIB}$ is the first European standard (EN50090 and EN 13321-1) ${ }^{40}$ international standard (ISO/IEC 14543-3) ${ }^{24}$ and Chinese Standard (GB/Z 20965). This technology is used to control security systems, heating, ventilation, air conditioning, monitoring, alarming, water control, energy management, metering as well as household appliances and audio ${ }^{26}$.

$\mathrm{KNX} / \mathrm{EIB}$ is a multimedia protocol with which it is possible to send signals by a cable (BUS). The signals can be Power Line, RF, IR, Bluetooth, and this protocol accepts the Ethernet protocol. This signal can be sent by a PC, domotic devices or home devices such as switches. A configuration of a system is presented in Figure 11.

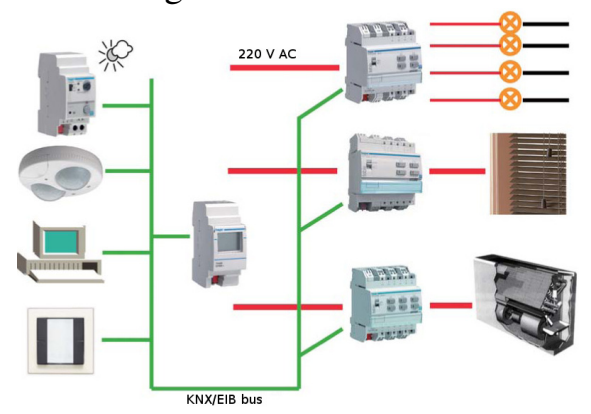

Fig. 11. KNX/EIB bus.

The proposed algorithms allow us to know the position of the laser spot and the device that the user wants to use. These algorithms have been combined with a Linux based KNX/EIB domotic system developed by Werntges ${ }^{43}$. The two techniques in conjunction allow us to obtain a whole system enabling orders to be sent to devices selected by users with the laser pointer.

We have used a KNX/EIB domotic system for experiments. It is composed of a power supply, a switch device, a USB interface and the KNX/EIB bus. The power supply is used to feed the various domotic components. The power supplies are essential for a specific, robust and efficient communication bus. The switch device allows us to connect the different home devices which will be turned on/off by it. Finally, the USB interface enables communication between the PC and the KNX/EIB installation. The USB interface is simply connected to the $\mathrm{KNX} / \mathrm{EIB}$ bus and then connected to the PC. The USB interface is automatically detected by the PC operating system and installed. Figure 12 shows the domotic panel that we use to simulate a real home environment. The system sends the information by a usb cable which allows us to turn on/off the de- 
vices selected by the handicapped person, using the laser pointer.

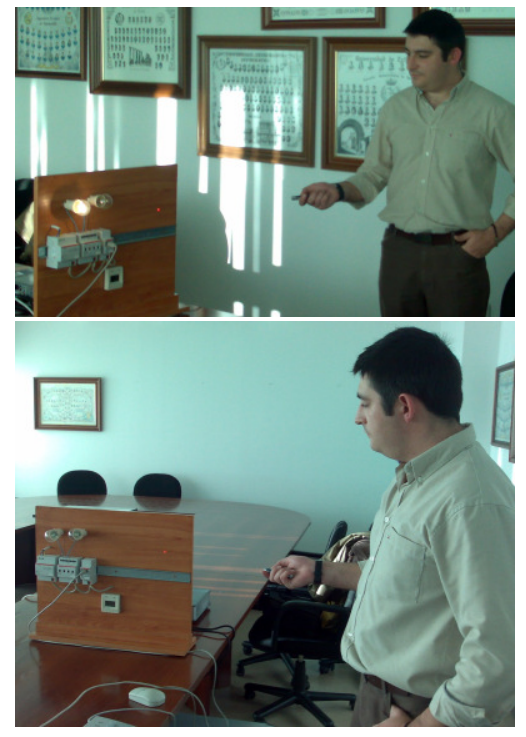

Fig. 12. Domotic panel

The whole system allows the users to indicate the active zones and the home devices to control, by means of the family tool. In the second step, by using a laser pointer, a disabled or elderly person can indicate the environment device which he wants to use. Thanks to the KNX/EIB domotic system, the computer can send the necessary orders to turn on/off the device selected by the user to the domotic panel.

\subsection{Experimental Set-Up}

The set of 990 images used to evaluate the different techniques has been randomly divided into 5 subsets with $20 \%$ of the images for each one. Applying a 5fold cross-validation method we obtained 5 different sets of images with 792 images for training and 198 images for test. Six different seeds have been used for each executions of the GA, using the 5 subsets. The results are the average of the 30 results obtained for each experiment.

We will compare the proposed method to the previous approaches described in Section 2.3. Table 4 shows a resume of the techniques used to detect the laser spots, using the set of images for training and test explained above.

The TM+DU algorithm uses the same configuration presented in ${ }^{14}$. The parameters for the methods $T M+F R B S_{\text {expert }}$ and $T M+F R B S_{\text {tuned } G A}$ were selected according to the recommendation of the authors in ${ }^{16}$ and ${ }^{15}$. Notice that in the $T M+F R B S_{\text {tuned } G A}$ and $T M+F R B S_{R G B-t u n e d G A}$ algorithm, the number of evaluations used by the GA in the tuning process was 50,000. Thus, the values of the input parameters considered by our proposal are shown in the next:

- Evaluations $=50,000$

- Population size $=200$

- Cross probability = 0.6

- Mutation probability $=0.1$

- Parameter $\mathrm{d}=0.35$

\subsection{Experiments and Analysis of Results}

In this section we present the results obtained by the studied techniques. Two different studies have been performed. First of all, we present a study of the previous approaches compared to the proposed algorithm, $T M+F R B S_{R G B-t u n e d G A}$, by using the big data set of the 990 images applying the 5-fold crossvalidation method in all techniques. Then, in order to show the benefits of the new approach, in the Subsection 4.3.2 we analyze the effects of the color consideration when compared to other alternatives.

\subsubsection{Comparing with the previous approaches}

In this subsection we present the study of the previous approaches with the complete data set of images. Table 5 presents the results for all the techniques used in this paper. We can see that the classic technique, $T M+D U$, shows good results. However, the FRBS design from an expert and tuned by the GA does not present the good results shown in 15 with respect to the classic approach when the new set of cases is considered. Finally, when we use the color influence, the best results are obtained. 
Table 4. Techniques used in the experiments

\begin{tabular}{llll}
\hline Ref & Method & Year & Description \\
\hline 14 & $T M+D U$ & 2008 & Template Matching plus Dynamic Umbralization \\
16 & $T M+F R B S_{\text {expert }}$ & 2010 & Template Matching plus FRBS design from Expert's Experience \\
15 & $T M+F R B S_{\text {tuned } G A}$ & 2010 & $T M+F R B S_{\text {expert }}$ tuned by GA \\
Our proposal & $T M+F R B S_{R G B-t u n e d G A}$ & & Template Matching plus automatic FRBS design including \\
& & & RGB color and tuned by GA \\
\hline
\end{tabular}

Table 5. Results of the proposed approach and comparison with the previous techniques

\begin{tabular}{llcccccc}
\hline System & & \multicolumn{2}{c}{$\begin{array}{c}\text { General Success } \\
\text { Rate }\end{array}$} & \multicolumn{2}{c}{$\begin{array}{c}\text { Success Rate in Images } \\
\text { with Laser Spot }\end{array}$} & \multicolumn{2}{c}{$\begin{array}{c}\text { Success Rate in Images } \\
\text { without Laser Spot }\end{array}$} \\
\hline & & Training & Test & Training & Test & Training & Test \\
\hline$T M+D U$ & Average & $83.48 \%$ & $83.54 \%$ & $67.86 \%$ & $68.13 \%$ & $98.32 \%$ & $98.36 \%$ \\
& St Deviation & 0.01206 & 0.04705 & 0.01575 & 0.05405 & 0.00481 & 0.01695 \\
$T M+F R B S_{\text {expert }}$ & Average & $78.59 \%$ & $78.59 \%$ & $66.21 \%$ & $66.52 \%$ & $90.35 \%$ & $90.30 \%$ \\
& St Deviation & 0.012293 & 0.049174 & 0.018454 & 0.0667703 & 0.008211 & 0.032265 \\
$T M+F R B S_{\text {tunedGA }}$ & Average & $75.80 \%$ & $74.97 \%$ & $51.44 \%$ & $50.51 \%$ & $98.91 \%$ & $98.35 \%$ \\
& St Deviation & 1.819656 & 5.035607 & 3.595273 & 6.846273 & 0.333988 & 1.345300 \\
$T M+F R B S_{R G B-\text { tuned } G A}$ & Average & $87.04 \%$ & $85.94 \%$ & $73.71 \%$ & $72.75 \%$ & $99.70 \%$ & $98.74 \%$ \\
& St Deviation & 0.711530 & 3.264756 & 1.118490 & 4.795984 & 0.219497 & 1.256093 \\
\hline
\end{tabular}

\subsubsection{On the Influence of the Color Consideration}

We can see that the FRBS developed by the expert does not produce good results. It is necessary to study whether this problem is due to a particular adaptation that the previous approaches present with respect to the sort set of examples considered. To do this, we must study whether it is necessary to improve the TM coefficient precision and/or to relearn the RB and/or readjust the MFs. In this way, we will perform different experiments on $T M+$ $F R B S_{\text {expert }}$ and two different possibilities, namely $T M+F R B S_{W M}$ and $T M+F R B S_{W M-\text { tuned } G A}$ :

- With $T M+F R B S_{\text {expert }}$ we will analyze the influence of the $T M$ precision parameter, by using the initial RB generated by the expert ${ }^{16}$ but trying different values for the TM precision parameter, ZMNCC (see Section 2.3.2). Table 6 shows the results of this system.

- $T M+F R B S_{W M}$ analyzes the influence of the RB generated for the examples, since it could not consider the new cases in the new, more representative set of examples. In this case, the RB is automatically generated by the Wang and Mendel al- gorithm ${ }^{59}$ to consider the new example data. Table 7 shows the results of this system.

- Finally, $T M+F R B S_{W M-t u n e d G A}$ consists of the previous system, $T M+F R B S_{W M}$, tuned by the GA proposed in ${ }^{15}$, once the new rules have been learned. Table 8 shows the results of this system with the $T M$ precision parameter fixed at 0.55 which has been shown to be the most effective.

The systems presented above take into account the new set of images. However, these new systems do not obtain better results than the classic system, $T M+D U$. For this reason, it is necessary to analyze the new characteristics of the laser spot images. From the results in Table 5, it has been shown that the color is a significant characteristic to correctly detect the laser spot on an image. As we can see in Figure 7, an image with a laser spot has a special color near to green saturation. However, an image with similar morphology, which is not a laser spot image, does not have this green color saturation. For these reasons, we have developed a new approach including three new variables to define the color of the image analyzed. A new FRBS has been developed using a color image parameter and has been 
tuned by a GA, namely $T M+F R B S_{R G B-t u n e d G A}$. This system has the best results, as we can see in Table 5.

The $T M+F R B S_{R G B-t u n e d G A}$ system analyzes the morphology and the color of the image sent by the video camera, obtaining the best results and almost completely eliminating the false offs.

\section{Conclusions}

In this paper a new hybrid technique between TM and FRBS is presented to detect a laser spot in a home environment. Thanks to a system developed in conjunction with a standard domotic system, we try to provide a more user-friendly and less expensive home device control environment, since this system works without expensive video cameras with filters and complex laser pointers. This system has been tested with a set of 990 real images, where the au-

Table 6. Analysis of the influence of the $T M$ precision parameter for $T M+F R B S_{\text {expert }}$ (i.e., by using initial RB generated by experts in ${ }^{16}$ ) when color is still not considered.

ZMNCC: Zero Mean Normalize Cross Correlation

\begin{tabular}{ccccccc}
\hline $\begin{array}{c}\text { Precision of ZMNCC } \\
\text { in TM algorithm }\end{array}$ & \multicolumn{2}{c}{$\begin{array}{c}\text { General Success } \\
\text { Rate }\end{array}$} & \multicolumn{2}{c}{$\begin{array}{c}\text { Success Rate in Images } \\
\text { with Laser Spot }\end{array}$} & \multicolumn{2}{c}{$\begin{array}{c}\text { Success Rate in Images } \\
\text { without Laser Spot }\end{array}$} \\
\hline & Training & Test & Training & Test & Training & Test \\
\hline 0.45 & $67.88 \%$ & $67.88 \%$ & $83.41 \%$ & $83.53 \%$ & $53.16 \%$ & $53.34 \%$ \\
0.50 & $71.72 \%$ & $71.62 \%$ & $76.36 \%$ & $76.56 \%$ & $67.14 \%$ & $67.29 \%$ \\
0.55 & $78.59 \%$ & $78.59 \%$ & $66.21 \%$ & $66.52 \%$ & $90.35 \%$ & $90.30 \%$ \\
0.65 & $77.68 \%$ & $77.68 \%$ & $64.13 \%$ & $64.40 \%$ & $90.55 \%$ & $90.49 \%$ \\
0.70 & $77.47 \%$ & $77.47 \%$ & $63.30 \%$ & $63.59 \%$ & $90.94 \%$ & $90.88 \%$ \\
\hline
\end{tabular}

Table 7. Analysis of the influence of the $T M$ precision parameter for $T M+F R B S_{W M}$ (i.e., $\mathrm{RB}$ is automatically generated by the Wang and Mendel algorithm to consider the new example data) when color is still not considered.

ZMNCC: Zero Mean Normalize Cross Correlation

\begin{tabular}{ccccccc}
\hline $\begin{array}{c}\text { Precision of ZMNCC } \\
\text { in TM algorithm }\end{array}$ & \multicolumn{2}{c}{$\begin{array}{c}\text { General Success } \\
\text { Rate }\end{array}$} & \multicolumn{2}{c}{$\begin{array}{c}\text { Success Rate in Images } \\
\text { with Laser Spot }\end{array}$} & \multicolumn{2}{c}{$\begin{array}{c}\text { Success Rate in Images } \\
\text { without Laser Spot }\end{array}$} \\
\hline & Training & Test & Training & Test & Training & Test \\
\hline 0.45 & $72.80 \%$ & $73.23 \%$ & $53.93 \%$ & $53.60 \%$ & $90.67 \%$ & $92.05 \%$ \\
0.50 & $72.50 \%$ & $72.42 \%$ & $53.15 \%$ & $51.86 \%$ & $90.82 \%$ & $92.05 \%$ \\
0.55 & $75.30 \%$ & $74.55 \%$ & $50.62 \%$ & $48.77 \%$ & $98.72 \%$ & $99.03 \%$ \\
0.60 & $74.97 \%$ & $74.14 \%$ & $49.73 \%$ & $47.94 \%$ & $98.92 \%$ & $99.03 \%$ \\
0.65 & $73.84 \%$ & $73.23 \%$ & $47.39 \%$ & $46.08 \%$ & $98.92 \%$ & $99.03 \%$ \\
0.70 & $73.38 \%$ & $72.73 \%$ & $46.45 \%$ & $44.99 \%$ & $98.92 \%$ & $99.03 \%$ \\
\hline
\end{tabular}

Table 8. TM $+F R B S_{W M-t u n e d G A}$ results on models obtained with $\mathrm{ZMNCC}$ fixed to 0.55 by applying a genetic tuning

\begin{tabular}{lcccccc}
\hline & \multicolumn{2}{c}{$\begin{array}{c}\text { General Success } \\
\text { Rate }\end{array}$} & \multicolumn{2}{c}{$\begin{array}{c}\text { Success Rate in Images } \\
\text { with Laser Spot }\end{array}$} & \multicolumn{2}{c}{$\begin{array}{c}\text { Success Rate in Images } \\
\text { without Laser Spot }\end{array}$} \\
\hline & Training & Test & Training & Test & Training & Test \\
\hline Average & $79.69 \%$ & $78.42 \%$ & $59.97 \%$ & $58.92 \%$ & $98.44 \%$ & $97.01 \%$ \\
Standard Deviation & 1.826459 & 3.859593 & 3.585886 & 5.724642 & 0.463948 & 1.329090 \\
\hline
\end{tabular}


thors attempt to simulate the real operating conditions of the system.

The new hybrid technique presented in this paper examines the morphology and color influence on laser spot images together, thus obtaining a better image analysis system for the laser spot. This system is able to almost completely remove the false offs and it increases the success rate in images with laser spots, for this reason, the general success rate is increased, obtaining a faster and more effective system.

We have presented an FRBS improved algorithm in combination with a laser pointer as a candidate to help disabled people to control home devices. It is particularly useful for remote acting on home devices as the user can reach any device in sight. Moreover, we think the results show the usefulness for any environment where devices are not easily reachable by the user, such as industrial operations.

\section{Acknowledgments}

This work has been supported by the Spanish Ministry of Education and Science under projects TIN2008-06681-C06-01 and National Nohnes project TIN2007-68083-C02-01, University of Extremadura, regional government Junta de Extremadura, Consejería de Economía-Comercio e Innovación and FEDER, project GRU09105.

\section{References}

1. E. Aguirre, A. González, “A Fuzzy Perceptual Model for Ultrasound Sensors Applied to Intelligent Navigation of Mobile Robots.", Applied Intelligence, 19(3), 171-187 (2003).

2. B.A. Ahlborn, D. Thompson, O. Kreylos,B. Hamann B. and O. Staadt, "A practical system for laser pointer interaction on large displays," VRST '05: Proceedings of the ACM symposium on Virtual reality software and technology, 106-109 (2005)

3. R. Alcalá, J. Alcalá-Fdez,M. Gacto, F. Herrera, "Improving fuzzy logic controllers obtained by experts: A case study in hvac systems," Applied Intelligence, 31(1), 15-30 (2009).

4. R. Alcalá, J. M. Benítez, J. Casillas, O. Cordón, R. Pérez, "Fuzzy Control of HVAC Systems Optimized by Genetic Algorithms.”, Applied Intelligence, 18(2), 155-177 (2003).
5. R. Alcalá, J. Casillas, O. Cordón, A. González, F. Herrera, "A genetic rule weighting and selection process for fuzzy control of heating, ventilating and air conditioning systems.", Engineering Applications of Artificial Intelligence, 18(3), 279-296 (2005).

6. F. Aldrich, "Smart Homes: Past, Present and Future," Inside the Smart Home, 17-39 (2003).

7. A. Bardossy, L. Duckstein, "Fuzzy Rule-Based Modeling With Application to Geophysical, Biological and Engineering Systems", CRC Press, (1995).

8. I. Basicevic, D. Kukolj, M. Popovic, "On the application of fuzzy-based flow control approach to High Altitude Platform communications.", Applied Intelligence, 1-12 (2009).

9. S. Borkowski, J. Letessier and J.L. Crowley, "Spatial Control of Interactive Surfaces in an Augmented Environment," Lecture Notes in Computer Science Springer, 3425, 228-244 (2005).

10. A.M. Brad, B. Rishi, N. Jeffrey, P.C. Hong, K. Dave, M. Robert and L.A. Chris, "Interacting at a distance: measuring the performance of laser pointers and other devices," CHI '02: Proceedings of the SIGCHI conference on Human factors in computing systems, 33-40 (2002).

11. M.S. Brown and W.K.H. Wong, "Laser pointer interaction for camera-registered multiprojector displays," IEEE International Conference on Image Processing, 913-916 (2003).

12. M. Chan, D. Estève, C. Escriba, and E. Campo, "A review of smart homes-present state and future challenges," Computer Methods and Programs in Biomedicine, 91(1), 55-81 (2008).

13. C.K. Charles, D.A. Cressel, N. Hai, J.T. Alexander and X. Zhe, "A point-and-click interface for the real world: laser designation of objects for mobile manipulation," HRI '08: Proceedings of the 3rd ACM/IEEE international conference on Human robot interaction, 241-248 (2008).

14. F. Chávez, F. Fernández, G. Olague, J. Llano, “An independent and non-intrusive laser pointer environment control device system," ICPS '08: Proceedings of the 5th international conference on Pervasive services, 37-46 (2008).

15. F. Chávez, F. Fernández, J. Alcalá-Fdez, R. Alcalá, F. Herrera, and G. Olague, "Genetic tuning of a laser pointer environment control device system for handicapped people with fuzzy systems," in IEEE International Conference on Fuzzy Systems (FUZZ-IEEE 2010), $1-8$ (2010).

16. F. Chávez, F. Fernández, R. Alcalá, J. Alcalá-Fdez, G. Olague, and F. Herrera, "Hybrid laser pointer detection algorithm based on template matching and fuzzy rule-based systems for domotic control inrealhome environments," Applied Intelligence, In Press, DOI: 10.1007/s10489-010-0268-6 (2011). 
17. F. Cincotti, D. Mattia, F. Aloise, S. Bufalari, G. Schalk, G. Oriolo, A. Cherubini, M.G. Marciani and F. Babiloni, "Non-invasive brain-computer interface system: Towards its application as assistive technology," Brain Research Bulletin, 75(6), 796-803 (2008).

18. O. Cordón, F. Herrera, A. Peregrín, "Applicability of the fuzzy operators in the design of fuzzy logic controllers.”, Fuzzy Sets and Systems 86(1), 15-41 (1997).

19. J. Davis and X. Chen, "Lumipoint: Multi-user laserbased interaction on large tiled displays," Displays, 23(5), 205-211 (2002).

20. D. Driankov, H. Hellendoorn, M. Reinfrank, "An introduction to fuzzy control (2nd ed.).", SpringerVerlag, London, UK, (1996).

21. R.M. Dufour, E.L. Miller and N.P. Galatsanos, “Template matching based object recognition with unknown geometric parameters," IEEE Transactions on Image Processing, 11(12), 1385-1396 (2002).

22. M.J. Gacto, R. Alcalá and F. Herrera, "A MultiObjective Evolutionary Algorithm for an Effetive Tuning of Fuzzy Logic Controllers in Heating, Ventilating and Air Conditioning Systems", Applied Intelligence, In Press, DOI: 10.1007/s10489-010-0264$\mathrm{x}(2011)$.

23. M. Goossens M, "The EIB system for home and building electronics," The EIB Handbook Series. The EIB Association, Brussels (1998).

24. International Electrotechnical Commission Portal, "http://www.iec.ch/"

25. L. Jiang, D. Liu and B. Yang, "Smart home research," Machine Learning and Cybernetics, 2004. Proceedings of 2004 International Conference on, 2, 659-663 (2005).

26. KNX Association, "http://www.knx.org/"

27. R. Kadouche1, B. Abdulrazak1, M. Mokhtari, S. Giroux 1 and H. Pigot, "Personalization and Multiuser Management in Smart Homes for Disabled People," International Journal of Smart Home, 3(1), 3948 (2009).

28. N.W. Kim, S.J. Lee, B.G. Lee and J.J. Lee, "Vision based laser pointer interaction for flexible screens," J.A. Jacko (ed.) Proceedings of the 12th international conference on Human-computer interaction: interaction platforms and techniques, 4551, 845-853 (2007).

29. C. Kirstein and H. Mueller, "Interaction with a projection screen using a camera-tracked laser pointer," Multi-Media Modeling Conference International, 191 (1998).

30. KNX/EIB, "http://www.knx.org"

31. M.E. Latoschik and E. Bomberg, "Augmenting a laser pointer with a diffraction grating for monoscopic 6dof detection," Journal of Virtual Reality and Broadcasting, 4(14), (2007).

32. C. Marie, E. Daniel, E. Christophe and C. Eric, "A review of smart homes-present state and future challenges," Computer Methods and Programs in Biomedicine, 91(1), 55-81 (2008).

33. S. Matveyev and M. Göbel, "Direct interaction based on a two-point laser pointer technique," A.P. Rockwood (ed.) International Conference on Computer Graphics and Interactive Techniques, 1-1 (2003).

34. J.Y. Oh and W. Stuerzlinger, "Laser pointers as collaborative pointing devices," Proceedings Graphics Interface, 141-150 (2002).

35. G. Olague, F. Fernández, C. Pérez and E. Lutton, "The infection algorithm: An artificial epidemic approach for dense stereo correspondence," Artificial Life, 12(4), 593-615 (2006).

36. J. Olsen, R. Dan and N. Travis, "Laser pointer interaction," CHI '01: Proceedings of the SIGCHI conference on Human factors in computing systems, 17-22 (2001).

37. R. Orpwood, C. Gibbs, T. Adlam, R. Faulkner and D. Meegahawatte, "The design of smart homes for people with dementiauser-interface aspects," Universal Access in the Information Society, 4(2), 156-164 (2005).

38. J. Park, C. Hsu and H. Arabnia, "Intelligent systems and smart homes," Information Systems Frontiers, 11(5), 481-482 (2009).

39. K. H. Park, Z. Bien, J.J. Lee, B.K. Kim, J.T. Lim, J.O. Kim, H. Lee, D.H. Stefanov, D.J. Kim, J.W. Jung, J.H. Do, K.H. Seo, C.H. Kim, W.G. Song and W.J. Lee, "Robotic smart house to assist people with movement disabilities," Autonomous Robots, 22(2), 183198 (2007).

40. Portal W3J, ’http://www.w3j.com/“

41. W.K. Pratt, "Digital Image Processing, Wiley, UK, (2001).

42. B. Shizuki, T. Hisamatsu, S. Takahashi and J. Tanaka, "Laser pointer interaction techniques using peripheral areas of screens," A. Celentano (ed.) Proceedings of the working conference on Advanced visual interfaces, 95-98. (2006).

43. H.W. Werntges, J. Neumann and V. Vinarski, "Controlling eib/knx devices from linux using usb," Tagungsband Konnex Scitific Conference., (2005).

44. B. Xiaojun, S. Yuanchun and C. Xiaojie, "upen: A smart pen-liked device for facilitating interaction on large displays," TABLETOP '06: Proceedings of the First IEEE International Workshop on Horizontal Interactive Human-Computer Systems, 160-168 (2006).

45. L.A. Zadeh, "Outline of a new approach to the analysis of complex systems and decision processes," IEEE Transactions on Systems, Man, and Cybernetics, SMC-3, 28-44 (1973).

46. L.A. Zadeh, "Is there a need for fuzzy logic?," Information Sciences, 178, 2751-2779 (2008).

47. L. Zhang, Y. Shi and J. Chen: "Drag and Drop by Laser Pointer: Seamless Interaction with Multiple 
Large Displays," Lecture Notes in Computer Science Springer, 4159, 12-20 (2006).

48. F. Herrera, M. Lozano, and J. L. Verdegay, "Tuning fuzzy logic controllers by genetic algorithms," International Journal of Approximate Reasoning, 12(3-4), 299-315 (1995).

49. D. Goldberg, "Genetic algorithms in search, optimization, and machine learning", Addison-Wesley Professional (1989).

50. J. Holland, "Adaptation in Natural and Artificial Systems". University of Michigan Press (1975).

51. O. Cordón, F. Herrera, F. Hoffmann, and L. Magdalena, "GENETIC FUZZY SYSTEMS. Evolutionary Tuning and Learning of Fuzzy Knowledge Bases", World Scientific (2001).

52. F. Herrera, "Genetic fuzzy systems: taxonomy, current research trends and prospects", Evolutionary Intelligence, 1(1), 27-46 (2008).

53. O. Cordón, F. A. C. Gomide, F. Herrera, F. Hoffmann, and L. Magdalena, "Ten years of genetic fuzzy systems: current framework and new trends", Fuzzy Sets and Systems, 141(1), 5-31 (2004).

54. N. Noury, G. Virone, P. Barralon, J. Ye, V. Rialle, and J. Demongeot, "New trends in health smart homes," Enterprise Networking and Computing in Healthcare Industry, 2003. Healthcom 2003. Proceedings. 5th International Workshop on, 118 - 127 (2003).
55. M. Nikolova, F. Meijs, and P. Voorwinden, "Remote mobile control of home appliances," Consumer Electronics, IEEE Transactions on, 49(1), 123 - 127 (2003).

56. J.-H. Do, H. Jang, S. H. Jung, J. Jung, and Z. Bien, "Soft remote control system in the intelligent sweet home," Intelligent Robots and Systems, 2005. (IROS 2005). 2005 IEEE/RSJ International Conference on, 3984 - 3989 (2005).

57. C. Kemp, C. Anderson, H. Nguyen, A. Trevor, and $\mathrm{Z}$. Xu, "A point-and-click interface for the real world: laser designation of objects for mobile manipulation," Proceedings of the 3rd ACM/IEEE international conference on Human robot interaction (HRI'08), 241248 (2008).

58. Y. W. Teng and W. J. Wang, "Constructing a userfriendly ga-based fuzzy system directly from numerical data," IEEE Trans. Syst., Man, Cybern., 34(5), 2060-2070 (2004).

59. L. X. Wang and J. M. Mendel, "Generating fuzzy rules by learning from examples," IEEE Trans. Syst., Man, Cybern., 22(6), 1414-1427 (1992).

60. L. X. Wang, "Adaptive Fuzzy Systems and Control. Design and stability analysis", Prentice-Hall, (1994).

61. E. Wilson, C. Karr, J. Bennett, "An Adaptive, Intelligent Control System for Slag Foaming.", Applied Intelligence, 20 (2), 165-177 (2004). 